\title{
Dimorfismo sexual e alometria ontogenética em Goyazana castelnaui (Crustacea, Brachyura)
}

\author{
Lucas Nunes da Silva (iD https://orcid.org/0000-0002-0327-0829 \\ Paulo Rogério de Souza Almeida https://orcid.org/0000-0002-0341-5530 \\ Renata Akemi Shinozaki-Mendes (1) https://orcid.org/0000-0002-1850-4763
}

Laboratório de Biologia Pesqueira, Unidade Acadêmica de Serra Talhada da Universidade Federal Rural de Pernambuco.

Av. Gregório Ferraz Nogueira, s/n CEP 56909-535 Serra Talhada, PE, Brasil.

Recebido 8 novembro 2017

Aceito 11 janeiro 2018

Publicado 11 June 2018

DOI 10.1590/1678-4766e2018008

\begin{abstract}
Sex dimorphism and ontogenetic allometry of Goyazana castelnaui (Crustacea, Brachyura). In crustaceans, studies of dimorphism using geometric morphometry approach are particularly appropriate due to the facility and accuracy in the identification of homologous landmarks. The aim of this study was identify the existence of sexual and age dimorphism of freshwater crab Goyazana castelnaui (H. Milne-Edwards, 1853), except for the dimorphism of the pleon, since it is an aspect intrinsic to the Brachyura. Were determined 10 anatomical landmarks for ventral view and 11 for the dorsal view. A covariance matrix was generated, containing factors related to the sexes and age groups of each sex, and the images were considered symmetrical. The canonical variate analysis (CVA) and discriminant analysis (DA) were performed with 999 permutations and as comparisons were made based on the Hotteling test $\left(\mathrm{T}^{2}\right)$ of the Procrustes distance. In the ventral view no variation was found in the form of juveniles and adults of females and males ( $p=0.4548, p=0.131$, respectively) based on the Procrustres distance. In the dorsal view, a separation was observed between the adult females and the other groups, indicating a noticeable modification of the cephalothorax volume for the allocation of the gonads.
\end{abstract}

KEYWORDS. Crustaceans, body shape, geometric morphometrics, cephalothorax, pleon.

RESUMO. Nos crustáceos, os estudos de dimorfismo sexual utilizando morfometria geométrica são particularmente adequados, devido à facilidade e acurácia na identificação dos marcos anatômicos. O objetivo do presente estudo foi identificar a existência de dimorfismo sexual e etário do caranguejo de água doce Goyazana castelnaui (H. Milne-Edwards, 1853), excetuando-se o dimorfismo do pléon, por tratar-se de um aspecto intrínseco aos Brachyura. Foram determinados 10 marcos anatômicos para vista ventral e 11 para a vista dorsal. Foi gerada uma matriz de covariância contendo fatores referentes aos sexos e aos grupos etários de cada sexo, sendo as imagens consideradas simétricas. A Análise de Variáveis Canônicas (CVA) e a análise discriminante (DA) foram realizadas com 999 permutações e as comparações foram feitas com base no teste de Hotteling $\left(\mathrm{T}^{2}\right)$ da distância de Procrustes. Para a vista ventral não foi encontrada nenhuma variação na forma entre jovens e adultos de fêmeas e machos $(p=0,4548 ; p=0,131$, respectivamente) com base na distância de Procrustes. Na vista dorsal, foi observada uma separação entre as fêmeas adultas e os demais grupos, indicando uma modificação notória do volume do cefalotórax para alocação das gônadas.

PALAVRAS-CHAVE. Crustáceos, forma do corpo, morfometria geométrica, cefalotórax, pléon.

Vários padrões no crescimento de crustáceos são adaptativos, sendo moldados por pressões seletivas que procuram maximizar a sobrevivência e o sucesso dos indivíduos (HARTNOLl \& Gould, 1988), podendo ser causadas por diversos fenômenos biológicos, o que leva a diferentes tipos de alometria. Uma destas é a alometria ontogenética, relacionada à variação da forma e tamanho de um organismo ao longo do seu desenvolvimento (KLINGENBERG, 1996). A variação corpórea pode se dar devido ao dimorfismo sexual, ou seja, diferenças morfológicas que estão relacionadas principalmente à seleção sexual (HARTNOLL, 1974) ou pela seleção natural (SHINE, 1989; FaIRBAIRN, 1997). Nos decápodos, o crescimento alométrico de diferentes porções corporais se mostra profundamente relacionado a mudanças em suas funções e especialização ao longo de sua ontogênese (HARTNOLL, 1978).

De acordo com PÉrez (1929), em Brachyura várias modificações relacionadas à maturidade podem ser observadas ao longo do seu desenvolvimento. Essa característica é amplamente conhecida pelas variações na forma e no tamanho do pléon nas fêmeas e do própodo dos quelípodos nos machos (Masunari \& Swiech-Ayoub, 2003). O campo da morfometria diz respeito a métodos que descrevem e analisam estatisticamente as variações de formas como um resultado do crescimento, tratamento experimental ou evolução (Rohlf \& Marcus, 1993). Segundo Moraes (2003), por meio da morfometria geométrica é possível 
descrever e localizar facilmente as regiões de mudanças na forma e, sobretudo, de construir e reconstituir graficamente estas diferenças, aumentando assim o poder discriminante, identificando diferenças morfológicas mínimas nas estruturas corporais despercebidas pela morfometria tradicional (BARRÍA et al., 2011).

De modo geral, técnicas de morfometria geométrica em crustáceos são utilizadas para identificar diferenças e variações significativas no formato e no tamanho de estruturas corpóreas, no intuito de entender questões evolutivas, ecológicas e relações intra- e interespecíficas (Rosemberg, 2002; GIRI \& Collins, 2004; RuFino et al., 2004; GIRI \& LOY, 2008; TUMELERO \& ForNel, 2014; AlENCAR et al., 2014; DiAwOL et al., 2015; MAROCHI et al., 2016; MAROCHI et al., 2017).

O caranguejo de água doce Goyazana castelnaui $(\mathrm{H}$. Milne-Edwards, 1853) pertence à Trichodactylidae (MELO, 2003), tem registro nos Estados do Pará, Maranhão, Tocantins, Pernambuco, Sergipe, Bahia, Goiás, Mato Grosso do Sul, São Paulo e Piauí (LimA-JúNIOR et al., 2008), ocorrendo também nas bacias dos rios Araguaia/Tocantins, Xingu, São Francisco, alto Paraguai e alto Paraná (Melo, 2003) e nas bacias costeiras do Maranhão e Sergipe (MAGALHÃES \& TÜRKAY, 1996). Apesar de apresentar ampla distribuição, as informações sobre $G$. castelnaui ainda são escassas na literatura e parte dos trabalhos existentes enfatiza a sua distribuição e ocorrência. Sendo assim, uma vez que adaptações morfológicas dos organismos refletem suas relações ecológicas (KARR \& JAMES, 1975), compreender a variação existente na sua forma (sexual e etária) auxiliará no entendimento de aspectos ainda desconhecidos para a espécie, como a morfologia funcional, a macroevolução, a seleção sexual, a biologia evolutiva, além de elucidar questões relacionadas à relação trófica como a predação.

Desta forma, objetiva-se aplicar, pela primeira vez, a técnica de morfometria geométrica para identificar a existência de dimorfismos sexual e etário (jovens e adultos) na vista dorsal (cefalotórax) e ventral (pléon) em indivíduos de G. castelnaui.

\section{MATERIAL E MÉTODOS}

Área de coleta. Foram capturados 98 indivíduos em um trecho do rio Pajeú, no município de Floresta, Pernambuco $\left(08^{\circ} 36^{\prime} 02^{\prime} \mathrm{S}, 38^{\circ} 34^{\prime} 05^{\prime} \mathrm{W}\right)$ (Fig. 1), no período entre julho de 2014 e outubro de 2015 . O município está localizado na mesorregião do São Francisco Pernambucano e microrregião do Sertão de Itaparica, cuja altitude é de aproximadamente 315,5 metros (CPRM, 2005). Os exemplares foram coletados no período noturno, com auxílio de puçás e feixes de luz.

Procedimento laboratorial. Os organismos foram crioanestesiados e posteriormente sexados com base na forma do pléon (triangular nos machos e semicircular nas fêmeas) (Melo, 2003). A classificação dos indivíduos quanto ao estado de maturação foi determinada a partir do grau de coloração do cefalotórax, onde os indivíduos adultos apresentam coloração vinho intenso e os jovens

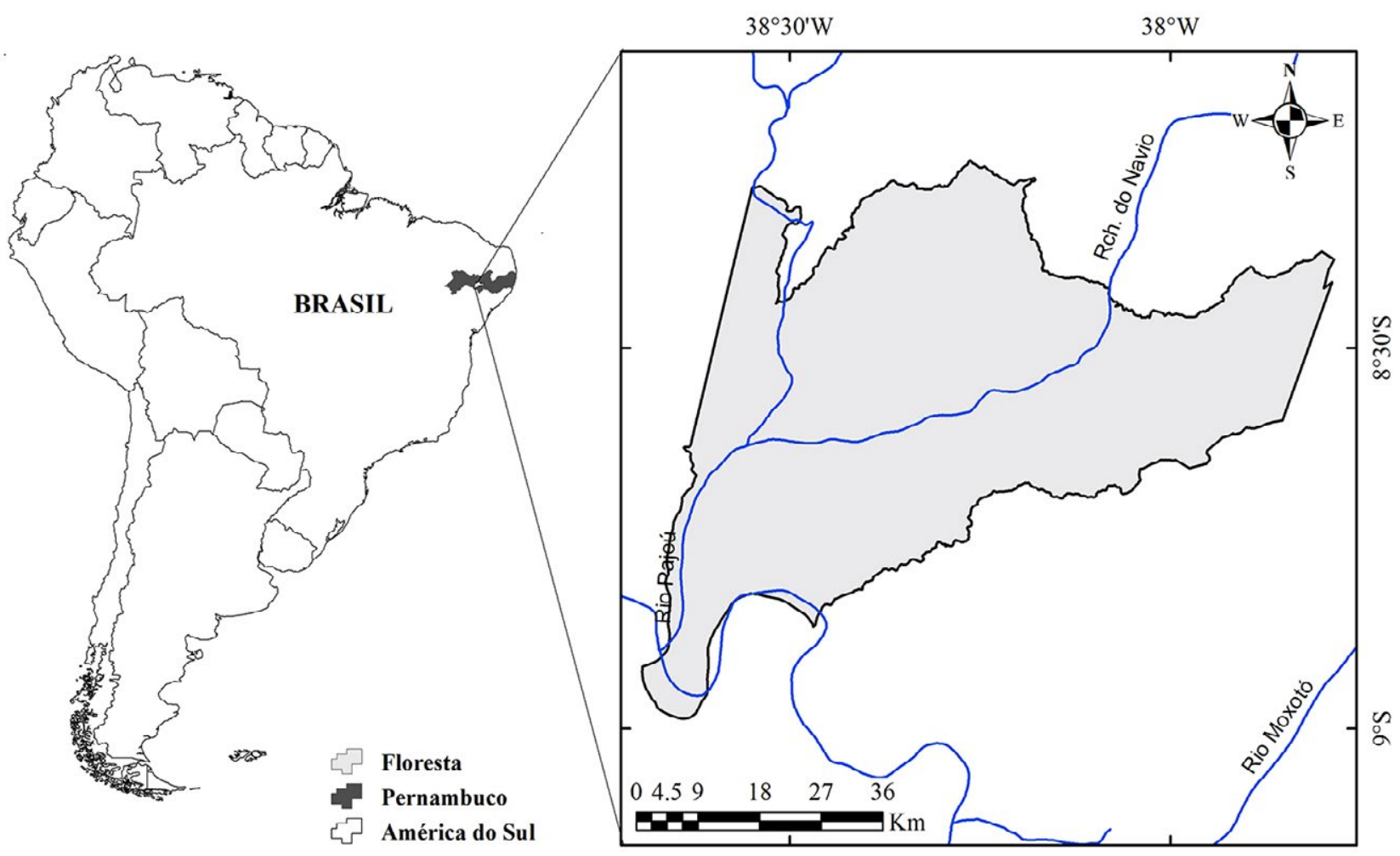

Fig. 1. Localização do município de Floresta (PE), Brasil, onde foi realizada a amostragem dos exemplares de Goyazana castelnaui (H. Milne-Edwards, 1853), no período entre julho de 2014 e outubro de 2015. 
bege-acinzentados, além de análises macro- e microscópica do trato reprodutor. Com auxílio de paquímetro (precisão de $0,01 \mathrm{~mm}$ ), foi mensurada a largura do cefalotórax (LC) na extremidade dos espinhos laterais.

Obtenção das variáveis morfométricas. Foram fotografados 63 exemplares machos (jovens $=18$ e adultos $=45$ ) e 35 exemplares fêmeas (jovens $=15$ e adultos $=$ 20) para as análises morfométricas. Os espécimes foram fotografados individualmente com auxílio de câmera digital paralela ao plano horizontal acoplado a um tripé, com lente de distância focal de $50 \mathrm{~mm}$.

Para determinar a forma do pléon, foram selecionados dez marcos anatômicos bidimensionais (vista ventral) e 11 marcos anatômicos bidimensionais para determinação da forma da carapaça (vista dorsal), para ambos os sexos (Fig. 2). Os marcos foram distribuídos nos pontos análogos e digitalizados com o programa TPSDig, versão 1.39 (RoHLF, 2006). Os marcos anatômicos foram classificados em função do grau de confiabilidade quanto à homologia de acordo com FORNEL \& ESTRELA (2012).

Foram realizadas análises para a vista dorsal (quatro grupos: fêmeas e machos, jovens e adultos). Em virtude do dimorfismo sexual do pléon ser um aspecto intrínseco aos Brachyura, as análises da vista ventral foram realizadas separadamente para machos e fêmeas (jovens $\mathrm{x}$ adultos). Uma Análise Generalizada de Procrustes (GPA) foi utilizada para realizar a sobreposição das coordenadas dos marcos anatômicos pelo centroide (centro de massa de uma configuração). Posteriormente, o tamanho do centroide de cada configuração foi escalonado para um valor mínimo, os marcos anatômicos correspondentes foram ajustados pelo método de quadrados mínimos e as diferenças que não estão relacionadas com a forma, tais como: escala, posição e orientação das configurações foram removidas (ADAMS et al., 2004; RohlF \& Marcus, 1993; BooksteIn, 1996).
Análises estatísticas. Foi realizada uma Análise de Componentes Principais (PCA) sobre a matriz de resíduos da GPA e os escores utilizados como novas variáveis de forma, para avaliar a ocorrência de dimorfismo sexual na forma. Foi gerada uma matriz de covariância, contendo fatores referentes aos sexos e aos grupos etários de cada sexo (Fêmea Jovem - FJ, Fêmea Adulta - FA, Macho Jovem - MJ e Macho Adulto - MA). A análise de variáveis canônica (CVA) foi realizada com 999 permutações. Para esta análise foi utilizada a Distância de Procrustes (Dist. Proc.) para comparar os grupos, onde maiores valores correspondem ao grau de separação existente entre os grupos. Diferenças entre as formas foram testadas através de Análise de Função Discriminante (DA) em conjunto com o teste de permutação, no intuito de calcular as percentagens de classificação e realizar uma validação cruzada entre os grupos (VISCOSI \& CARDINI, 2011). Com auxílio do programa MorphoJ foram geradas funções da Flexão de Placas Finas (Thin-plate Splines Functions) entre jovens e adultos, machos e fêmeas (KLINGENBERG, 2008), no intuito de mapear as deformações em uma grade de forma entre os sexos.

Para avaliar possíveis diferenças estatísticas de tamanho, foi utilizado o tamanho do centroide, definido como a raiz quadrada da soma dos quadrados da distância de cada marco do centroide (média de todas as coordenadas) (Bookstein, 1991). Para testar a alometria da forma em relação ao tamanho do centroide, foi realizada uma regressão multivariada dos escores da regressão das coordenadas de Procrustes em função do tamanho do centroide, considerando os quatro grupos (FA, FJ, MA, MJ); posteriormente, os resultados foram comparados utilizando 999 permutações $(\mathrm{p}<0,05)$.

Por fim, para avaliar possíveis diferenças estatísticas no tamanho da carapaça entre machos, fêmeas, jovens e adultos (quatro grupos analisados separadamente), inicialmente foi
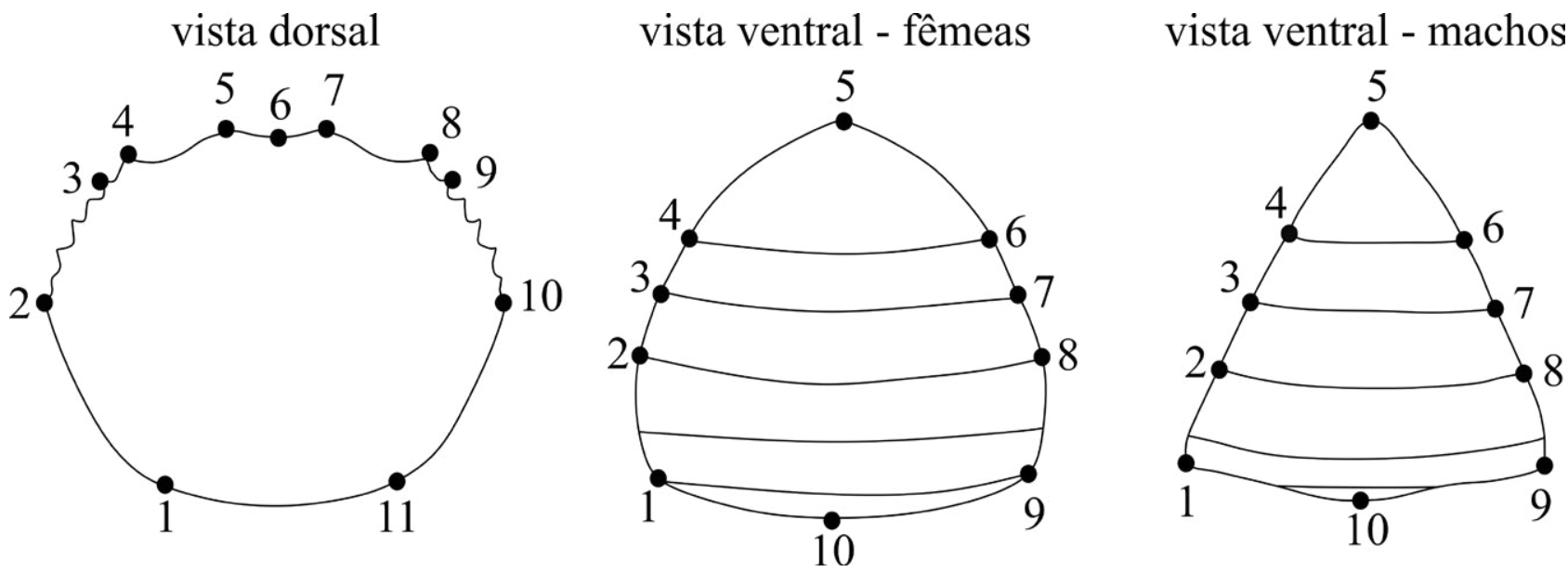

Fig. 2. Descrição dos marcos anatômicos utilizados na morfometria geométrica da vista dorsal e ventral de Goyazana castelnaui (H. Milne-Edwards, 1853). Vista dorsal: 1, margem póstero-lateral esquerda da carapaça; 2 e 10, extremidade do espinho lateral; 3 e 9, extremidade dos segundos dentes pós-orbital; 4 e 8, extremidade dos dentes pós-orbital; 5, 6, 7, margem frontal da carapaça; 11, margem póstero-lateral direita da carapaça. Vista ventral: 1 e 9, II somito abdominal; 2 e 8 , IV somito abdominal; 3 e 7; V somito abdominal; 4 e 6 , VI somito abdominal; 5 , ápice do telson; 10, base do pléon (porção ventral). 
testada a normalidade dos erros da distribuição (teste de Shapiro-Wilk) e a homocedasticidade das variâncias (teste de Bartlett) (ZAR, 2010). Em seguida, foi aplicado o teste de comparação entre as médias (ANOVA) seguido do teste de Tukey (ZAR, 2010).

\section{RESULTADOS}

Foram analisadas fêmeas jovens $(\mathrm{n}=15)$ com tamanhos de cefalotórax que variaram entre 11,8 e 47,7 mm (39,2 $\mathrm{mm}, 4$ $\mathrm{mm})$ e fêmeas adultas ( $\mathrm{n}=20)$, com variação de 33,9 a 50,2 $\mathrm{mm}(43,9 \pm 5 \mathrm{~mm})$. Nos machos essa variação foi de $10,5 \mathrm{a}$ $49,9 \mathrm{~mm}(42,7 \pm 3,1 \mathrm{~mm})$ para os jovens $(\mathrm{n}=18)$ e de $34,4 \mathrm{~mm}$ a $54,4 \mathrm{~mm}(44,6 \pm 3,7 \mathrm{~mm})$ para os adultos $(\mathrm{n}=45)$.

Análise da vista ventral para fêmeas e machos (analisados separadamente). Na análise de componentes principais (PCA), os três primeiros componentes apresentaram explicação na variação da forma de 90,03\% e 82,52\% para fêmeas e machos, respectivamente.

Na Análise de Variáveis Canônicas (CVA), as fêmeas (jovens $\mathrm{x}$ adultas) foram consideradas iguais, com base na distância de Procrustes ( $p=0,4548)$. Apesar da semelhança estatística observada para a CVA, as fêmeas apresentaram um deslocamento notório nos marcos 3, 5, 7 e 10 na transição de jovens para adultas. O marco 5 é responsável pela variação no comprimento do telson, enquanto que os marcos 3 e 7 representam a largura e o marco 10 a inserção anterior do pléon (Fig. 3).

Nos machos (jovens x adultos), não foi observado diferença entre os grupos $(p=0,131)$. Os indivíduos não apresentaram variação da forma (Fig. 3) e mostraram uma alocação correta de $67,44 \%$ e 58,12\%, em relação à análise discriminante e à validação cruzada, respectivamente.
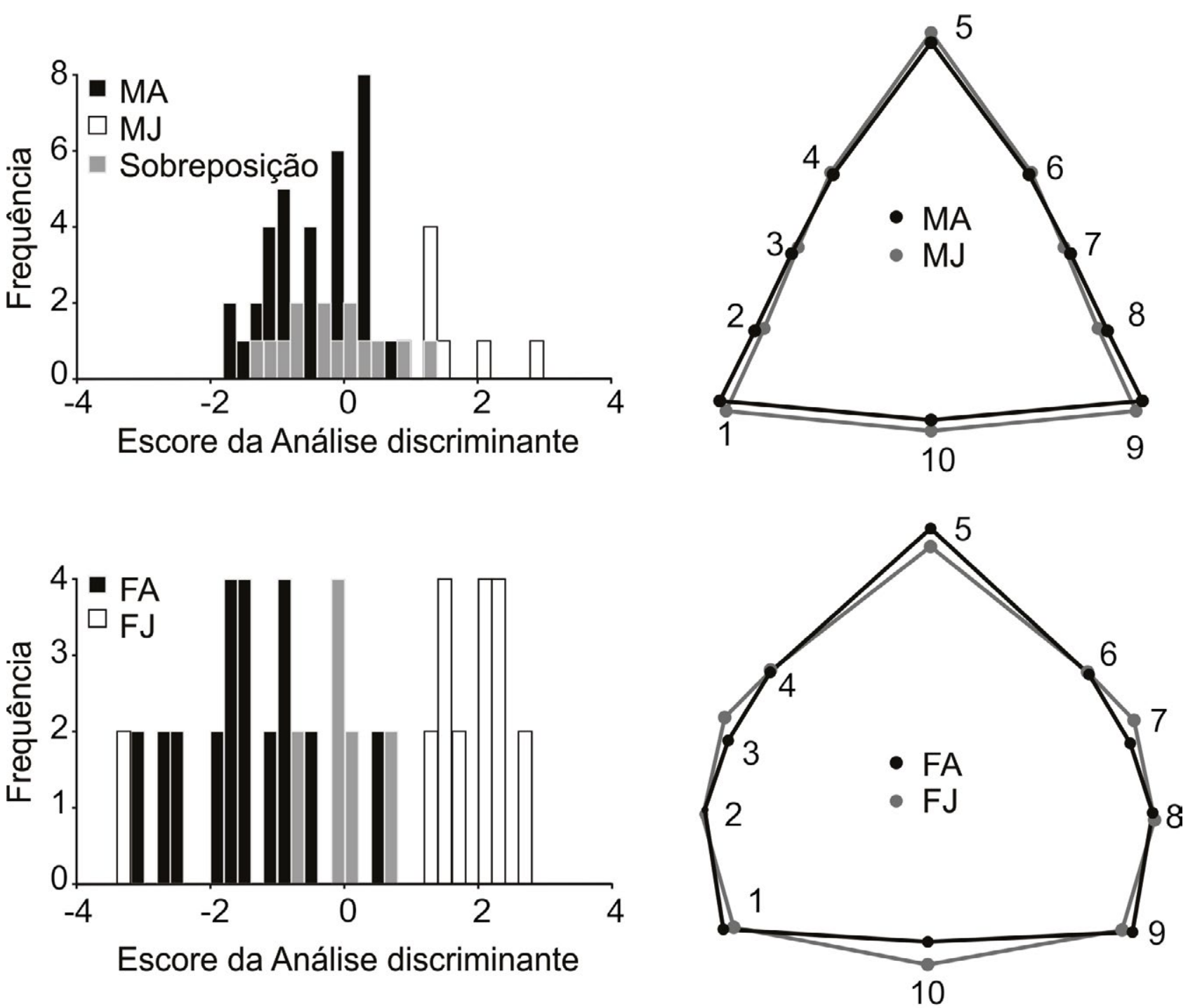

Escala: 3,0

Fig. 3. Gráficos de separação de grupo para machos (M) e fêmeas (F) jovens (J) e adultos (A) na análise discriminante (esquerda) e variação na vista ventral (direita) na comparação entre jovens e adultos de Goyazana castelnaui (H. Milne-Edwards, 1853) capturados no município de Floresta, PE, Brasil no período de julho de 2014 a outubro de 2015. 
Análise da vista dorsal. De acordo com a percentagem de variância, calculados por ordem decrescente de importância, os três primeiros componentes totalizaram um montante de explicação de variação na forma $83,03 \%$. Ao realizar a análise de variável canônica (CVA), foi observada uma separação com base na Dist. Proc $(p<0,001)$ apenas para as fêmeas adultas dos demais grupos (Fig. 4).

Com base na Análise Discriminante (DA) entre pares de grupo com permutação, o grupo com maior distância foi FA-MJ, seguido de FA-MA e FA-FJ, apresentando um percentual de classificação correta entre 80 e 95\% (Tab. I).

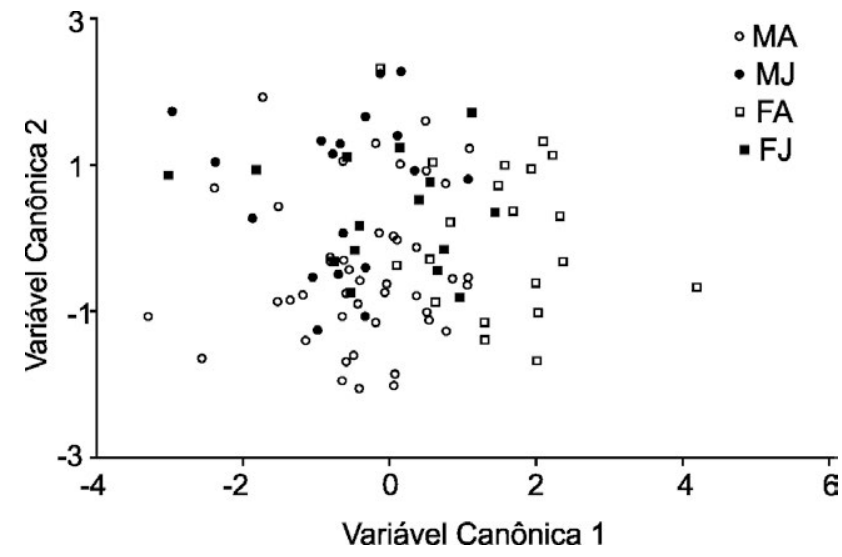

Fig. 4. Análise de variável canônica da vista dorsal de machos e fêmeas, jovens e adultos de Goyazana castelnaui (H. Milne-Edwards, 1853) capturados no município de Floresta, PE, Brasil no período de julho de 2014 a outubro de 2015 .

Tab. I. Resultado da análise discriminante da vista dorsal para interações par a par de machos e fêmeas, jovens e adultos de Goyazana castelnaui (H. MilneEdwards, 1853), capturados no município de Floresta, PE, Brasil no período de julho de 2014 a outubro de 2015 (FA, fêmea adulta; FJ, fêmea jovem; MA, macho adulto; MJ, macho jovem; Dist. Proc, distância de Procrustes; $\mathrm{p}<$, P- centroide valor; $(\% \mathrm{~A})$, alocação correta; $(\% \mathrm{C})$, validação cruzada.

\begin{tabular}{lccccc}
\hline Grupos & $\mathrm{P}$ & $\mathrm{D}^{2}$ & $\mathrm{P}<$ & $(\% \mathrm{~A})$ & $(\% \mathrm{C})$ \\
\hline FA-MJ & 0,034 & 2,989 & $<0,001$ & $95 \%$ & $90 \%$ \\
FA-MA & 0,030 & 2,099 & $<0,001$ & $85 \%$ & $70 \%$ \\
FA-FJ & 0,029 & 2,017 & 0,017 & $80 \%$ & $70 \%$ \\
MA-MJ & 0,012 & 1,240 & 0,071 & $80 \%$ & $73,33 \%$ \\
FJ-MA & 0,009 & 1,147 & 0,206 & $66,67 \%$ & $40 \%$ \\
FJ-MJ & 0,137 & 1,076 & 0,636 & $60 \%$ & $60 \%$ \\
\hline
\end{tabular}

Tanto o dimorfismo sexual como a alometria ontogenética foram fortemente relacionados à variação nos marcos anatômicos 1, 2 e seus homólogos 10 e 11 (Fig. 5), em que as fêmeas adultas apresentaram a margem lateral e posterior mais curta que os juvenis de ambos os sexos.

A análise do tamanho do centroide permitiu inferir que houve diferenças significativas para a vista dorsal $(\mathrm{p}=0,0002)$ devido à amplitude e variação de tamanhos observados nos valores médios de cada grupo. Quando analisado o valor médio do tamanho do centroide, diferenças notáveis foram observadas entre jovens e adultos $(\mathrm{FJ}=0,65 \pm 0,063$;
$\mathrm{FA}=0,57 \pm 0,09 ; \mathrm{MJ}=0,61 \pm 0,05 ; \mathrm{MA}=0,55 \pm 0,06$ ). Ao correlacionar os escores da regressão da PCA com o tamanho do centroide, não se observou nenhuma tendência $(\mathrm{p}=0,9450)$, embora possam ser observados apenas indivíduos jovens com centroide de até 0,5 e apenas adultos com centroide superior a 0,7 .

\section{DISCUSSÃO}

Alometria ontogenética. Neste estudo, nota-se que a variação apresentada na forma do pléon das fêmeas de Goyazana castelnaui não foi evidenciada na CVA. No entanto, tomando como base os marcos anatômicos selecionados, pode-se evidenciar graficamente variação na forma. As fêmeas adultas apresentaram pléon com o telson mais comprido, quando comparadas com o pléon das fêmeas jovens, corroborando com Shinozaki-Mendes \& Lessa (2017), que observou diferenças morfométricas na vista ventral de indivíduos jovens e adultos do Callinectes danae, capturado no Canal de Santa Cruz, onde as fêmeas adultas apresentaram variações quando comparadas com os outros grupos. Em adição, essa variação foi observada por Castiglioni \& Negreiros-Fransozo (2004), ao utilizarem técnicas de morfometria linear para determinar as dimensões do corpo que melhor indicam a maturidade de Uca rapax (Smith, 1870).

Uma base abdominal mais larga (marcos 1 e 9) pode ser justificada pela disponibilidade de parte de suas energias para a reprodução e durante o tempo em que as fêmeas incubam os ovos nos pleópodos (ADIYODI \& ADIYODI, 1970; HARTNOLL, 1985), comportamento característico da maioria dos decápodos límnicos (LóPEZ-GRECo et al., 2004). Entretanto, o estreitamento da porção dos marcos 3 e 7 apresentada no presente trabalho não foi relatada por outros autores em seus estudos. Notoriamente foi observado um maior abaulamento do pléon, aspecto que não é identificado no ângulo de captação das imagens, mas que pode ser percebido durante o manuseio dos animais e que justifica o deslocamento supracitado, bem como o deslocamento do marco 10.

Para os machos de G. castelnaui, os resultados encontrados não apresentaram variações morfológicas entre adultos e jovens, com relação à vista ventral. Isso ocorre devido à estrutura ser utilizada apenas para a sustentação e proteção dos dois pares de pleópodos responsáveis pela transferência do esperma durante o acasalamento como mostrado por CASTIGLIONI \& NEgREIROS-Fransozo (2004), não se evidenciando uma necessidade biológica que justifique a variação na sua forma.

Dimorfismo sexual. Ao analisar dorsalmente $G$. castelnaui, foi observado que existe variação da forma entre as fêmeas adultas e os demais grupos, em que as fêmeas adultas apresentam aumento da região posterior da carapaça, corroborando com TREvisAn et al. (2012), que encontrou em Aegla marginata Bond-Buckup \& Buckup, 1994 fêmeas mais largas na região posterior da carapaça. Uma vez que a mesma variação também ocorreu ao comparar fêmeas 

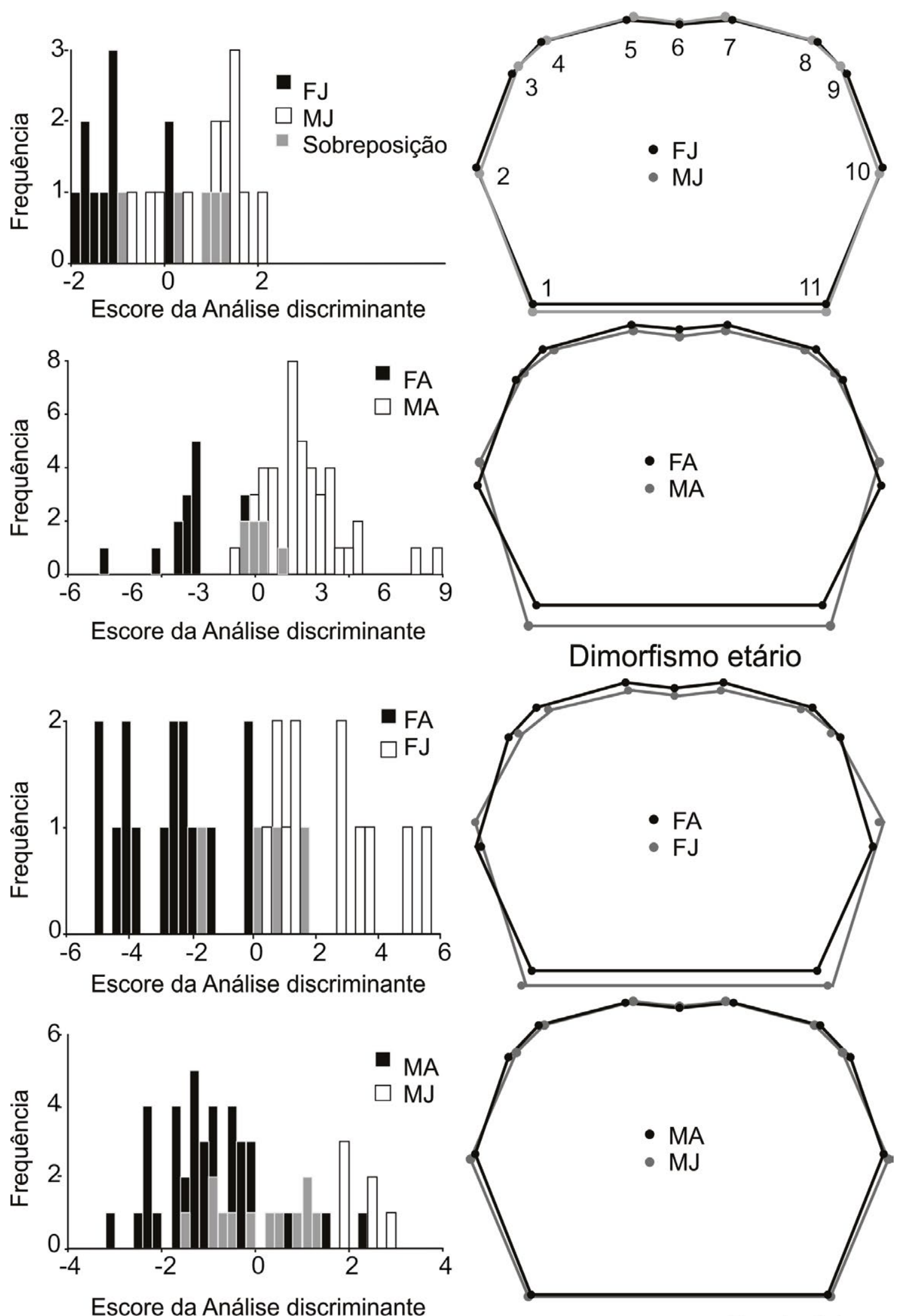

Escala: 3,0

Fig. 5. Gráfico de alocação de indivíduos quanto ao grupo para machos (M) e fêmeas (F) jovens (J) e adultos (A) na análise discriminante (esquerda) e variação na vista dorsal na comparação entre sexos e grupos etários de Goyazana castelnaui (H. Milne-Edwards, 1853) capturados no município de Floresta, PE, Brasil no período de julho de 2014 a outubro de 2015. 
jovens e adultas, e não apenas entre fêmea e macho, fica claro que não se trata da variação descrita por RuFino et al. (2004), ao analisarem as diferenças existentes entre machos e fêmeas do Liocarcinus depurator (Linnaeus, 1758), onde machos apresentaram a carapaça mais larga com dentes mais conspícuos, tratando-se de uma variação de tamanho, e não da forma, por AlENCAR et al. (2014) ao verificarem o dimorfismo sexual do Ucides cordatus (Linnaeus, 1763), que observou que as fêmeas apresentam a porção lateral do cefalotórax reduzida e por MAROCHI et al. (2016), que determinou o dimorfismo sexual na forma e no tamanho da carapaça em uma população de $H$. pudibundus apresentaram resultados onde as fêmeas exibiram a margem lateral posterior menos larga que os machos.

Provavelmente, há um aumento do volume interno do cefalotórax para acomodar as gônadas, que passam a ser volumosas dando um formato mais abaulado e, consequentemente, promovendo o deslocamento dos marcos. Esta variação nas fêmeas de G. castelnaui pode surgir como uma estratégia reprodutiva, estando ligada à sua maturidade fisiológica, otimizando assim o esforço reprodutivo (Сово \& Fransozo, 2005).

A similaridade encontrada na forma dos demais grupos pode ser causada pela combinação de diversos fatores, tais como o estado nutricional, a disponibilidade de alimentos e genética, conforme discutido por TzENG (2004) e Anastasiadou et al. (2009). Giesel (1972) e FAIrbairn \& Preziosi (1994) acreditam que a ausência de diferença estatística no tamanho da carapaça entre os sexos pode ser explicada em termos de vários fatores, incluindo a migração diferencial e/ou mortalidade entre os sexos e o isolamento geográfico. Estes fatores podem agir em conjunto com a seleção natural e sexual para produzir o tamanho da semelhança observada entre os sexos, podendo ainda essa variação estar relacionada ao número amostral.

Estatisticamente a análise do tamanho do centroide para avaliar a variação da forma entre os indivíduos de $G$. castelnaui reforça a existência de diferenças significativas baseada na observação da região dorsal. Em concordância, MARCHIORI et al. (2014), ao estudarem o tamanho do centroide para comparar a variação na forma da carapaça dos indivíduos de populações da Aeglia longirostri, observaram diferenças significativas de tamanho do centroide entre populações estudadas, divergindo de Trevisan et al. (2012), que compararam a forma da carapaça da Aegla marginata, não encontrando nenhuma diferença relevante quando se trata do tamanho do centroide.

Para G. castelnaui ainda não existem estudos que apontem a causa da similaridade na forma ou tamanho; no entanto, o isolamento geográfico, possíveis semelhanças nas taxas de mortalidade entre sexos e a biologia da espécie podem ser apontados como fatores classificatórios para esta variável, uma vez que os locais de distribuição destes indivíduos os deixam expostos a esses fatores. Embora tenha sido verificada diferença do tamanho do centroide, ao correlacionar os escores da regressão da PCA não se observou nenhuma tendência dos pontos. Essa dispersão pode estar relacionada ao baixo número amostral, não sendo ainda possível correlacionar aos gastos energéticos ou padrões.

Foi observado que o tamanho populacional da espécie nas diversas localidades do rio Pajeú é bastante reduzido, acarretando em uma baixa amostragem e dificultando a interpretação de algumas análises. Entretanto, por se tratar de uma espécie com raras informações populacionais, acreditase que, mesmo com um número baixo de exemplares, os resultados encontrados constituem informações valiosas para o seu conhecimento.

De modo geral, esta é a primeira contribuição que trata da morfometria geométrica do G. castelnaui, sendo necessário um investimento significativo para que essa e outras populações, em diversas regiões, sejam estudadas e seja possível uma melhor compreensão das funções adaptativas relacionadas às estruturas corporais da espécie.

\section{REFERÊNCIAS}

Adams, D. C.; Rohlf, F. J. \& Slice, D. E. 2004. Geometric morphometrics: ten years of progress following the 'revolution'. Italian Journal of Zoology 28:5-16.

AdiYodi, K. G. \& AdiYodi, R. G. 1970. Endocrine control of reproduction in Decapoda Crustacea. Biological Review 45:121-165.

Alencar, C. E. R. D.; Lima-Filho, P. A.; Molina, W. F. \& Freire, F. A. M. 2014. Sexual Shape Dimorphism of the Mangrove Crab Ucide scordatus (Linnaeus, 1763) (Decapoda, Ucididae) Accessed through Geometric Morphometric. The Scientific World Journal 1:1-8.

Anastasiadou, C.; Liasko, R. \& LeOnardos, I. D. 2009. Biometric analysis of lacustrine and riverine populations of Palaemonetes antennarius (H. Milne Edwards, 1837) (Crustacea, Decapoda, Palaemonidae) from north-western Greece. Limnologica 39:244-254.

Barría, E. M.; Sepúlveda, R. D. \& JARA, C. G. 2011. Morphologic variation in Aegla leach (Decapoda: Reptantia: Aeglidae) from CentralSouthern Chile: interspecific differences, sexual dimorphism, and spatial segregation. Journal of Crustacean Biology 31:231-239.

Bookstein, F. L. 1991. Morphometric Tools for Landmark Data: Geometry and Biology. Cambridge University Press, London. 435p.

Bookstein, F. L. 1996. Standard formula for the uniform shape component in landmark data. In: Marcus, L. F.; Corti, M.; LoY, A.; NAYlor, G. J. P. \& Slice, D. E. eds. Advances in morphometrics. New York, Plenum Press, p. 153-168.

Castiglioni, D. S. \& Negreiros-Fransozo, M. L. 2004. Comparative analysis of the relative growth of Uca rapax (Smith) (Crustacea, Ocypodidae) from two mangroves in São Paulo, Brazil. Revista Brasileira de Zoologia 21:137-144.

CoBo, V. \& Fransozo, A. 2005. Physiological maturity and relationships of growth and reproduction in the red mangrove crab Goniopsis cruentata (Latreille) (Brachyura, Grapsidae) on the coast of São Paulo, Brazil. Revista Brasileira de Zoologia 22:219-223.

CPRM. 2005. Serviço Geológico do Brasil. Projeto cadastro de fontes de abastecimento por água subterrânea. Diagnóstico do município de Floresta, Estado de Pernambuco. In: Beltrão, B. A.; Mascarenhas, J. C.; Miranda, J. C.; Souza Júnior, L. C.; Galvão, M. J. T. G. \& Pereira, S. N. eds. Diagnóstico do município de Floresta. Recife, CPRM/PRODEEM. 28p.

Diawol, V. P.; Giri, F. \& Collins, P. A. 2015. Shape and size variations of Aegla uruguayana (Anomura, Aeglidae) under laboratory conditions: A geometric morphometric approach to the growth. Iheringia, Série Zoologia 105:76-83.

FAIRBAIRN, D. J. 1997. Allometry for sexual dimorphism: pattern and process in the coevolution of body size in males and females. Annual Reviews in Ecology and Sistematics 28:659-687.

Fairbairn, D. J. \& Preziosi, R. F. 1994. Sexual selection and evolution of allometry for sexual size dimorphism in the water strider, Aquarius remigis. The American Naturalist 144:101-118. 
ForNel, R. \& Estrela, P. C. 2012. Morfometria geométrica e a quantificação da forma dos organismos. In: MARINHO, J. R.; HePP, L. U. \& ForNeL, R. eds. Temas em Biologia: Edição comemorativa aos 20 anos do Curso de Ciências Biológicas e aos 5 anos do PPG-Ecologia da URI Campus de Erechim. Erechim, EDIFAPES, p. 101-120.

GiesEL, J. T. 1972. Sex ratio, rate of evolution, and environmental heterogeneity. American Naturalist 106:380-387.

GIRI, F. \& Collins, P. A. 2004. A geometric morphometric analysis of two sympatric species of the family Aeglidae (Crustacea, Decapoda, Anomura) from the La Plata basin. Italian Journal of Zoology 71:85-88.

GIRI, F. \& Loy, A. 2008. Size and shape variation of two freshwater crabs in Argentinian Patagonia: The influence of sexual dimorphism, habitat, and species interactions. Journal of Crustacean Biology 28:37-45.

HARTNOLL, R. G. 1974. Variation in growth patterns between some secondary sexual characters in crabs. Crustaceana 27:131-136.

HaRTNOLL, R. G. 1978. The determination of relative growth in Crustacea. Crustaceana 34:281-293.

HARTNOLL, R. G. 1985. Growth, sexual maturity and reproductive output. $I n$ : WENNER, A. M. ed. Crustacean Issues: Factors in adult growth. Rotterdam, Balkema, p. 101-128

HaRTNOLL, R. G. \& Gould, P. 1988. Brachyuran life history strategies and the optimization of egg production. Symposia of the Zoological Society of London 59:1-9.

KARR, J. R. \& JAMES, F. C. 1975. Eco-morphological configurations and convergent evolution in species and communities. In: CoDY, M. L. \& DiAmOND, J. M. Ecology and evolution of communities. Cambridge, Harvard University Press, p. 191-258.

KlingenberG, C. P. 1996. Multivariate allometry. In: Marcus, L. F.; Corti, M.; Loy, A.; NaYlor, G. J. P. \& Slice, D. E. eds. Advances in morphometrics. New York, Plenum Press, p. 23-49.

Klingenberg, C. P. 2008. Software MorphoJ. Manchester, Faculty of Life Sciences, University of Manchester, UK. Disponível em <www. flywings.org.uk>.

Lima-Júnior, T. B.; Carvalho, J. B.; Santos, J. N.; Cruz, A. C. \& Leite, J. R. S. A. 2008. Novas ocorrências de Goyazana castelnaui (H. MILNEEDWARDS, 1853) (Crustacea, Brachyura, Trichodactylidae), para o estado do Piaú, Brasil. Boletim do Laboratório de Hidrobiologia 21:31-34.

López-Greco, L. S.; Viau, V.; Lavolpe, M.; Bond-Buckup, G. \& Rodriguez, E. M. 2004. Juvenile hatching and maternal care in Aegla uruguayana (Anomura, Aeglidae). Journal of Crustacean Biology 24:309-313.

MAgAlHÃES, C. \& TÜRKAY, M. 1996. Taxonomy of the neotropical freshwater crab family Trichodactylidae. I. The generic system with description of some new general (Crustacea: Decapoda: Brachyura). Senckenbergiana Biologica 75:63-95.

Marchiori, A. B.; Bartholomei-Santos, M. L. \& Santos, S. 2014. Intraspecific variation in Aegla longirostri (Crustacea: Decapoda: Anomura) revealed by geometric morphometrics: evidence for ongoing speciation? Biological Journal of the Linnean Society 112:31-39.
Marochi, M. Z.; Masunari, S. \& Schubart, C. D. 2017. Genetic and Morphological Differentiation of the Semiterrestrial Crab Armases angustipes (Brachyura: Sesarmidae) along the Brazilian Coast. Biological Bulletin 232:30-44.

Marochi, M. Z.; Trevisan, A.; Gomes, F. B. \& Masunari, S. 2016. Dimorfismo sexual em Hepatus pudibundus (Crustacea, Decapoda, Brachyura). Iheringia, Série Zoologia 106:e2016003.

Masunari, S. \& Swiech-Ayoub, B. P. 2003. Crescimento relativo em Uca leptodactyla Rathbun (Crustacea, Decapoda, Ocypodidae). Revista Brasileira de Zoologia 20:487-491.

Melo, G. A. S. 2003. Manual de identificação dos crustáceos decápodes de água doce do Brasil. São Paulo, Edições Loyola. 430p.

Moraes, D. A. 2003. Morfometria geométrica e a "revolução na morfometria" localizando e visualizando mudanças na forma dos organismos. Bioletim 3:1-5.

Pérez, C. 1929. Sur l'appareil d'accrochage de l'abdomen authorax chez les decapodes brachyoures. Comptès Rendús 186:461-463.

RoHLF, F. J. 2006. tpsDig, digitize landmarks and outlines, version 2.10. New York, Department of Ecology and Evolution, State University of New York at Stony Brook.

RoHLF, F. J. \& Marcus, L. F. 1993. A revolution in morphometrics. Trends in Ecology and Evolution 8:129-132.

RosemberG, M. S. 2002. Fiddler crab claw shape variation: a geometric morphometric analysis across the genus Uca (Crustacea: Brachyura: Ocypodidae). Biological Journal of the Linnean Society 75:147-162.

Rufino, M.; Abelló, P. \& Yule, A. B. 2004. Male and female carapace shape differences in Liocarcinus depurator (Decapoda, Brachyura): an application of geometric morphometric analysis to crustaceans. Italian Journal of Zoology 71:79-83.

SHINE, R. 1989. Ecological causes for the evolution of sexual dimorphism: a review of the evidence. The Quarterly review of Biology 64:419-461.

Shinozaki-Mendes, R. A. \& LesSA, R. 2017. Ontogenetic trajectories in Callinectes danae (Crustacea: Brachyura): sex and age polymorphism. Journal of the Marine Biological Association of the United Kingdom. Published online: 30 October 2017, pp. 1-8.

Trevisan, A.; Marochi, M. Z.; Costa, M.; Santos, S. \& Masunari, S. 2012. Sexual dimorphism in Aegla marginata (Decapoda: Anomura). Nauplius 20:75-86.

Tumelero, G. W. \& ForNel, R. 2014. Variação morfológica nas quelas de três espécies do gênero Aegla Leach, do sul do Brasil. Vivências 10:250-267.

Tzeng, T. D. 2004. Stock identification of sword prawn Parapenaeopsis hardwichii in the East China Sea and Taiwan Strait inferred by morphological variation. Fisheries Science 70:758-764.

VISCOSI, V. \& CARDINI, A. 2011. Leaf morphology, taxonomy and geometric morphometrics: a simplified protocol for beginners. Plos One 6:1-20.

ZAR, J. H. 2010. Biostatistical Analysis. 5ed. Upper Saddle River, Pearson Prentice-Hall. 944p. 